Trusting Labels: A Matter of Numbers?

Liebi Thomas

02-01

February 2002

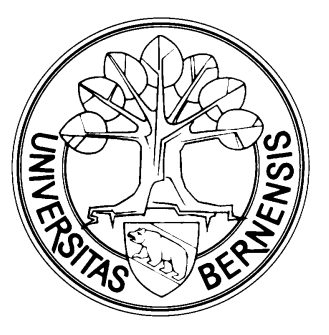

Universität Bern

Volkswirtschaftliches Institut

Gesellschaftstrasse 49

3012 Bern, Switzerland

Tel: $41(0) 316314506$

Web: www-vwi.unibe.ch 


\title{
Trusting Labels: A Matter of Numbers?
}

\author{
Thomas Liebi* \\ University of Bern \\ February 2002
}

\begin{abstract}
Labelling is an increasingly popular way to deal with the problem of non-observability of quality inherent in the consumption of credence goods. I present a model in which the number of labelled products a monopolist offers serves as a signal for the non-observable endogenous quality. An increase in the number of labelled products increases the risk of losing consumer trust by increasing the possibility of detecting wrong labels. This lowers the incentive to produce low quality in the first place.
\end{abstract}

Keywords: credence goods, labels, product quality, consumer trust. JEL-Classifications: L15, D82, M37

\footnotetext{
*Universitaet Bern, Volkswirtschaftliches Institut, Abteilung fuer Wirtschaftstheorie, Gesellschaftsstrasse 49, CH-3012 Bern, Switzerland, thomas.liebi@vwi.unibe.ch, http://www.vwi.unibe.ch/staff/ liebi/. I thank Esther Bruegger, Alain Egli, Winand Emons and Martin Wagner for helpful discussions.
} 


\section{Introduction}

It is well known that asymmetric information may cause serious market inefficiencies. In this context, credence goods present a special kind of problem: even after consumption the product's quality cannot be determined by the consumers. Many product attributes for which consumers are willing to pay a premium relative to the normal price have credence quality. To illustrate, consumers may care that the breakfast cereal was really produced organically, that food doesn't contain genetic modified organisms (GMO), that tuna was fished dolphin-friendly etc. Usually, the production of this kind of high quality goods is more costly than that of low quality ones. But if this is the case, consumers may doubt the quality specification given by the firm, since there's an evident incentive for the firm to produce low quality goods and sell them at high prices. This means, consumers may not be willing to pay the full premium, leading to lower profits for firms producing high quality goods, thereby lowering their incentive to invest in high quality which further increases consumers' doubts. In the end, this may lead to a non-provision of high quality goods although many people prefer consuming high quality and paying a premium. Thus, we may end up with an inefficient market outcome.

Several mechanisms have been proposed to circumvent this inefficient situation. Emons [1997 and 2001] analyzes the case where a customer does not know whether she really received the expert's service she was in need of. He shows that under certain conditions the expert provides honest services. A survey of credence goods and expert services is given by Dulleck and Kerschbamer [2001].

Shapiro [1982 and 1983] analyzes repeated buying of experience goods. He argues that expected higher future profits, called return to reputation, prevent firms from offering low quality at a premium price. Since product 
quality is not observable even after consumption, this mechanism doesn't apply with credence goods. A fast growing number of labelled goods, see for example Thøgersen [2000], indicates another way to try to convince potential customers of a firm's products' qualities. Golan, Kuchler and Mitchell [2001] discuss effects of food labelling and present some case studies and examples. By labelling whole product lines a firm may benefit from consumers' positive experience with other products bearing the same label. ${ }^{1}$ However, the firm also bears the risk that if one labelled product was found bad, consumers probably extend this negative experience to other products of the same line. This is the underlying idea in the articles of Wernerfelt [1988], Choi [1998] and Cabral [2000] about extending brands to new products. However, all the results are derived for the case of experience goods where the product's quality is observed after consumption. In addition, product quality is exogenous while in the underlying paper it will be the producer's choice.

In the following we shall see that although consumers cannot directly observe product quality, they may rely on the number of labelled products in a firm's product line as a signal for the possible loss in reputation that could occur if the firm tries to sell low quality credence goods at a premium price. This latter case does not have to mean malicious behavior by the firm but also includes carelessness in production, e.g. possible measurement errors concerning $\mathrm{GMO}^{2}$, and will be modelled as non-willingness to invest a certain amount in the production process.

The model is based on the idea that consumers mainly trust or mistrust firms

\footnotetext{
${ }^{1}$ If someone, for example, made good experiences with organically produced tea, she may choose to buy also coffee with the same label.

${ }^{2}$ In a recent case in Switzerland it was discovered that the fodder for animals within a meat-label-programme contained 17\% GMO which was way above the allowed 3\%. The reason was a measurement error indicating a GMO-share of only $1.7 \%$.
} 
or their labels rather than single products. So, if they lose faith in some labelled good, they will also doubt the quality of all other products with the same label. Empirical evidence supports the assumption that consumers extend bad news about a product's quality to other products with the same brand. For example, Sullivan [1990] shows that the 1986 sudden-acceleration incident with the Audi 5000 caused negative price effects for the Audi 4000 and the Audi Quattro as well.

We assume that after the production process but before the consumers' buying decision there is the possibility of detecting low quality goods in a firm's product line, be it through tests performed by a consumer protection organization, an employee publishing insider information, or otherwise. This will lead the firm to trade off higher profits by selling low quality at a high price thereby increasing the risk of losing the premiums on all labelled goods against lower but safer profits.

\section{The Model}

A monopolist facing homogeneous consumers can offer credence goods of either high or low quality. Given their preferences the consumers are willing to pay a premium $H>0$ for a high quality credence good and no premium for the low quality one. Before production takes place the firm decides whether it wants to invest an amount $c \geq 0$ per product type, like beer, wheat bread, blue T-shirts etc., in order to produce high quality or not. These costs may represent higher production costs, costly tests performed by the firm, monitoring costs etc. We assume $c$ to be the realization of a random variable $\tilde{c}$ which is distributed with density $\psi(\cdot)$ on $[0, \bar{c}]$. This distribution is known to both the monopolist and the consumers. The cost $c$ per product type, 
necessary to produce high quality, is exogenous and the firm only decides whether to invest or not. Thus, $c$ can be interpreted as an efficiency measure of a firm's production process.

Since the quality of a product is not directly observable by the consumer, the firm can put a label on a good, indicating high product quality, even if this is not true. It can thus try to get the premium without having to invest c. We will call such a label a "bad label", whereas a label on a high quality product is called a "good label".

In the following we will concentrate our analysis on the part of a firm's products that do bear a label. Let the firm's product line consist of $n$ different products, all of which bear a label. We will consider a short run situation meaning that the number of products is fixed at $n$, e.g., by capacity constraints, market potential etc. The monopolist is free to choose the quality of each product type independently of the other types, i.e., for how many product types he wants to invest $c .{ }^{3}$ The firm's choice variable therefore is the number of good labels among those $n$ labelled products. It will be modelled as the firm choosing the fraction $s \in[0,1]$ of good labels relative to $n$, the total number of labelled products.

After the production process, but before consumers buy the good, there is the possibility that bad labels are detected through testing, information leaks etc.

\footnotetext{
${ }^{3}$ For example, a firm might choose to offer high quality coffee but low quality potato chips where "quality" is always referring to the credence attribute.
} 
For each bad label there is a probability $q$, where $0<q<1$, of being detected. ${ }^{4}$

Note that we want to exclude errors in the detection process. In particular, it is not possible that a good label is found bad. Both the firm and the consumers are informed whether there was detection of any bad labels or not. After this, the monopolist sets the price for his products. We normalize the units of the different products such that the price, resp. the premium $H$ that consumers are willing to pay for a high quality good, is the same for each product group. Finally, consumers decide whether to buy the products or not. To keep matters simple we assume that firms produce only one unit of each product type, i.e., $n$ different labelled products altogether. ${ }^{5}$ Both the firm and the consumers are risk-neutral. Further on we assume that $\bar{c}<H{ }^{6}$

\subsection{Firm's Cost is Known to Consumers}

Let us first consider the case in which the premium $H$, the detection probability $q$ and the production costs $c$ are known to both monopolist and consumers.

The sequence of events is as follows:

Stage 1: The monopolist decides upon the fraction of good labels relative

\footnotetext{
${ }^{4}$ If $q=0$ no bad label is ever detected. The firm has no incentive to invest in good labels and - knowing this - consumers would not pay any premium. Otherwise, if $q=1$ every bad label would be detected but then we do not face a credence good problem, a case we want to exclude.

${ }^{5}$ Instead of doing so, we could also regard $H$ as the total revenue from a whole product group.

${ }^{6}$ This condition implies that even if the firm is of the least efficient type, there is a surplus from producing high quality.
} 
to the total number of labelled products, i.e., he chooses $s$.

Stage 2: Nature chooses whether there is detection of fraud or not, i.e., if at least one product with a bad label has been found. Consumers observe if there have been detected any bad labels or not and form their beliefs about the quality of the products. Let $\mu$ be the probability they assign to the product being of high quality.

Stage 3: The monopolist sets a price $P$ as a function of $H, q, n, c$, and $\mu$ per unit of product.

Stage 4: The consumers will buy the products or not. We assume that consumers will buy the products if they are indifferent.

Risk neutral consumers are willing to pay a price $P(\cdot)$ for each labelled good, where $P(\cdot)=\mu H+(1-\mu) 0$, with $\mu$ representing the probability consumers assign to a labelled product being of high quality.

The assumption that consumers trust or distrust a firm as a whole, resp. its labels, rather than single products, is modelled as the consumers reducing the price they are willing to pay for all labelled goods, if there have been any bad labels.

We will say that if consumers observe that at least one bad label has been detected, they reduce the price they are willing to pay for each labelled product by some amount $k$, where $0 \leq k \leq P(\cdot)$. The price reduction $k$ is endogenous and depends on the beliefs that consumers have about being offered high quality. In equilibrium the price reduction $k$ by a rational consumer is either 0 - if no bad label was detected and the number of labelled products reaches a certain value - or $P(\cdot)$ if at least one bad label was detected. Consumers know that if there has been no detection of fraud, this 
could be either because all labels are good or because, by chance, none of the bad ones was detected. Accordingly, they are willing to pay a price $P(\cdot)$ based an all the information they have.

Within the given framework there is a probability of $(1-q)^{n(1-s)}$ that no bad label is detected and the firm receives the price $P(\cdot)$ for each product it sells. With a probability of $1-(1-q)^{n(1-s)}$ at least one bad label is detected and the firm only gets $P(\cdot)-k$ per unit of the product. The firm maximizes expected profits

$$
\pi^{e}=(1-q)^{n(1-s)} n P(\cdot)+\left(1-(1-q)^{n(1-s)}\right) n[P(\cdot)-k]-n s c
$$

by choosing $s$ for a given $n$. We shall see that consumers are able to derive a threshold value for $n$ which we call $\bar{n}$, below which the firm will produce only low quality products. So, if consumers observe that the number of labelled products is smaller than $\bar{n}$, they will pay no premium for any good, even if there was no detection of any bad label. Therefore, equation (1) is only valid for a firm having $n \geq \bar{n}$, otherwise it will only produce low quality and receive a price of 0 for them.

If

$$
\frac{\partial \pi^{e}}{\partial s}=-(1-q)^{n(1-s)} n^{2} \ln (1-q) k-n c>0
$$

expected profits are increasing in $s$ and therefore in the number of good labels for a given number of labelled products.

The last inequality can be rewritten as

$$
-(1-q)^{n(1-s)} \ln (1-q) n k>c .
$$


Condition (2) tells us that in order to invest $c$ in one more product given $n$, thereby reducing the number of bad labels by one, marginal costs of doing so, $c$, have to be smaller than marginal benefits. These benefits consist of the reduction in the risk of detection over the whole product line, $-n(1-$ $q)^{n(1-s)} \ln (1-q)$, times the additional price the firm can get for a labelled product by avoiding the consumers' "punishment" $k$.

Note that given $n$, expected profits are convex ${ }^{7}$ in $s$ since

$$
\frac{\partial^{2} \pi^{e}}{\partial s^{2}}=n^{3} k(\ln (1-q))^{2}(1-q)^{n(1-s)}>0
$$

Therefore the optimal choice of $s$ is either 0 or 1 , i.e., the firm will either choose to have only bad or only good labels in its product line but no mixture of both. Accordingly, if the firm decides to bear the costs to produce high quality goods it will not risk the premia by also offering bad labelled products. On the other hand, if premia are low relative to costs and the detection probability is not too high, it will decide not to invest at all rather than just investing in a few high quality goods.

Given this binary choice, the firm just compares expected profits if it has only good labels with the case where it has only bad ones. The firm chooses to offer only high quality goods, i.e., $s^{*}=1$, if

$$
n P(\cdot)-n c \geq(1-q)^{n} n k+n P(\cdot)-n k
$$

or if

$$
\left(1-(1-q)^{n}\right) k \geq c .
$$

\footnotetext{
${ }^{7}$ Strictly convex if $k>0$.
} 
Since all the parameters in condition (3) are known to the consumers, they are able to derive the monopolist's optimal $s$, being 0 or 1 . Hence, they are able to derive the true quality of the labelled goods and choose to pay the corresponding price. Therefore, either $\mu^{\star}=1$ and $P(\cdot)=H$ for high quality, or $\mu^{\star}=0$, resp. $P(\cdot)=0$, for low quality products.

We see from condition $(3)$ that for any $q \in(0,1)$ there exists a certain number of labelled goods $\bar{n}$ such that the firm prefers $s=1$ to $s=0 \forall n \geq \bar{n}$. This shows that if the firm has a critical size, measured by the number of labelled products, it will behave honestly, meaning that it will not offer any bad labelled products.

Consumers know that the monopolist offers either only high or only low quality products. Accordingly, they will refuse to pay any premium at all if at least one bad label was detected, i.e., $k=P(\cdot)$. If condition (3) is satisfied for $k=P(\cdot)$, consumers know that all products are of high quality. Considering that in this case they are willing to pay $P(\cdot)=H$ and inserting this into condition (3), the critical $n$ can then easily be derived, since at $\bar{n}$ it is true that

$$
\left(1-(1-q)^{\bar{n}}\right) H=c .
$$

This leads to

$$
\bar{n}=\frac{\ln \left(1-\frac{c}{H}\right)}{\ln (1-q)} .
$$

Note that $c / H$ is smaller than one, given $\bar{c}<H$.

Let us check how changes in the parameter values affect this threshold value $\bar{n}$. 
(i) Changes in costs:

$$
\frac{\partial \bar{n}}{\partial c}=-\frac{1}{\ln (1-q)(H-c)}>0
$$

since $H>c$. The higher the firm's cost, the larger is the threshold value $\bar{n}$. If costs to produce high quality are increasing, there is an increasing incentive to produce low quality under a bad label. Thus, the threshold value $\bar{n}$ has to be larger, such that the higher risk to lose all the premia under bad labels offsets the incentive to produce low quality.

(ii) Changes in the achievable premium:

$$
\frac{\partial \bar{n}}{\partial H}=\frac{c}{\ln (1-q) H(H-c)}<0
$$

since $H>c$. The higher the premium consumers are willing to pay for a high quality good, the higher is the firm's incentive not to risk those premia by offering bad labels. Therefore, the number of labelled products can be smaller to guarantee high quality production.

(iii) Changes in the detection probability:

$$
\frac{\partial \bar{n}}{\partial q}=\frac{\ln (1-c / H)}{(\ln (1-q))^{2}(1-q)}<0
$$

The higher the probability of detection, the higher the risk to lose all the premia by offering bad labels. Therefore, with an increasing detection probability $q$, the threshold value $\bar{n}$ can be lower to still guarantee high quality production.

The above considerations are summarized in the following lemma. 
Lemma 1 If $c$ is known to the consumers, then there is a threshold value $\bar{n}$ for the number of labelled products above which consumers can be sure of getting high quality products. This threshold value increases with a firm's cost $c$, and decreases with the premium $H$ and the detection probability $q$.

Proposition 1 summarizes the equilibrium obtained in this section.

Proposition 1 Given the above defined consumer behavior and the fact that all the relevant parameters are known to the consumers, we find the following Bayesian equilibrium:

- if consumers observe that $n<\bar{n}$ as defined in equation (4), they expect only low quality products $\left(\mu^{\star}=0\right)$ and don't pay any premium even if there is no detection of a bad label. A monopolist with $n<\bar{n}$ will decide not to produce any high quality products and sets the price $P(\cdot)=0$.

- if consumers observe $n \geq \bar{n}$ and no detection of a bad label, they expect only high quality products $\left(\mu^{\star}=1\right)$ and pay the full premium $H$ per unit of the good. A monopolist with $n \geq \bar{n}$ will decide to produce only high quality products and sets the price $P(\cdot)=H$.

- if consumers observe the detection of at least one bad label, they expect only low quality products and don't pay any premium. A monopolist who suffered the detection of a bad label will set the price $P(\cdot)=0$.

Therefore, the number of labelled products is a signal for the quality of the firm's products.

\subsection{Firm's Cost is Unknown to Consumers}

In general, consumers do not know a firm's production process. Therefore, let us now consider the situation where consumers observe the parameters $q$, $H$, and $n$, but not $c$. Again, before deciding whether to buy the good or not, 
consumers learn if any bad labels were detected, although they do not know the exact number, resp. fraction of bad labels detected.

The sequence of events is as follows:

Stage 1: The monopolist learns his cost $c$ and chooses the fraction $s$ of good labels relative to the total number of labelled goods.

Stage 2: Nature chooses whether there is detection of fraud or not, i.e., if at least one product with a bad label has been found. Consumers observe if any bad labels were detected or not and form their beliefs about the quality of the products. Let $\mu$ be the probability they assign to the product being of high quality.

Stage 3: The monopolist sets a price $P$ as a function of $H, q, n, c$, and $\mu$ per unit of product.

Stage 4: The consumers will buy the products or not. We assume that consumers will buy the products if they are indifferent.

We still model consumer behavior such that they reduce the price they are willing to pay by some amount $k$ if any bad labels have been detected or they pay a price $P=\mu H+(1-\mu) 0$ per unit if no detection occurred. The difference to the previous section is that now $\mu$ is not a function of $c$. Given consumer behavior, the monopolist's expected profit is again given by equation (1), i.e.,

$$
\pi^{e}=(1-q)^{n(1-s)} n P(\cdot)+\left(1-(1-q)^{n(1-s)}\right) n[P(\cdot)-k]-n s c .
$$

Given this, the conclusions about the shape of expected profits made in the last section remain true. Most important is the fact that expected profits are 
still convex with respect to $s$, which leads the monopolist to a binary choice between $s=0$ and $s=1$. Accordingly, if consumers observe the detection of at least one bad label they will refuse to pay any premium on all the labelled goods, i.e., $k=P(\cdot)$. Knowing the distribution of $c$, the consumers can derive the probability that the monopolist will choose $s=1$. Let us call this probability, i.e., the probability that all the labels are good, $\rho(\cdot)$.

The game in extensive form is represented in figure 1.

\section{[Insert figure 1 about here]}

Consumers know that

- if any bad label is detected, i.e., the game reaches point $\mathrm{C}$, then all labels must be bad. This happens with an ex-ante probability of $(1-\rho(\cdot))(1-(1-$ $\left.q)^{n}\right)$,

- if there is no detection, then it is either because there are only good labels or because there are only bad labels but by chance none was detected. These two possibilities form an information set which is reached with an ex-ante probability of $\rho(\cdot)+(1-q)^{n}(1-\rho(\cdot))$.

In the first case, i.e., if any bad labels are detected, the consumers will not pay any premium and the monopolist sets the price $P(\cdot)=0$.

In the second case, i.e., if there is no detection, consumers will update their beliefs according to Bayes' rule.

They assign a probability of

$$
\mu^{\star}:=\frac{\rho(\cdot)}{\rho(\cdot)+(1-\rho(\cdot))(1-q)^{n}}
$$

to being in point A, i.e., that all labels are good, and a probability of 


$$
1-\mu^{\star}:=\frac{(1-\rho(\cdot))(1-q)^{n}}{\rho(\cdot)+(1-\rho(\cdot))(1-q)^{n}}
$$

to being in point B, i.e., that all labels are bad. Accordingly, they are willing to pay a price

$$
P(\cdot)=\mu^{\star} H+\left(1-\mu^{\star}\right) 0
$$

for the product in the case of no detection.

The monopolist's expected profits are therefore represented by

$$
\pi^{e}=(1-q)^{n(1-s)} n \mu^{\star} H-n s c .
$$

Given the firm's binary choice, it will compare expected profits under $s=1$ with those under $s=0$. It will choose to produce only high quality goods $\left(s^{\star}=1\right)$ if

$$
n \mu^{\star} H-n c \geq(1-q)^{n} n \mu^{\star} H
$$

or if

$$
\mu^{\star} H\left(1-(1-q)^{n}\right) \geq c
$$

From condition $(8)$ we can now derive $\rho(\cdot)$, the probability that only high quality goods are produced. Define

$$
\rho(\cdot):=\operatorname{Prob}\left(c \leq \mu^{\star} H\left(1-(1-q)^{n}\right)\right)
$$


To get further results, let us assume that $c$ is uniformly distributed between 0 and $\bar{c}$. For convenience, the left hand term of condition (8) will be called $A$ in the following.

For $\rho(H, q, n, \bar{c}) \neq 0{ }^{8}{\text { we } \text { get }^{9}}^{9}$

$$
\rho(H, q, n, \bar{c})=\left\{\begin{array}{cll}
\frac{H}{\bar{c}}-\frac{(1-q)^{n}}{\left(1-(1-q)^{n}\right)} & \text { if } & 0<A<\bar{c} \\
1 & \text { if } \quad A \geq \bar{c}
\end{array}\right.
$$

Accordingly, $\rho(H, q, n, \bar{c})$ is increasing if the number of labelled products $n$ increases, meaning that the probability of only high quality goods being produced is higher the more labelled products the firm has. Returning to equation (6) we see that in this case the price consumers are willing to pay is a positive function of the number of labelled products the firm has, ${ }^{10}$ with $P(q, H, c, n, \mu)$ converging to $H$ as $n$ goes to infinity.

This result is summarized in the following lemma:

Lemma 2 In the above defined framework the price consumers are willing to pay for a labelled credence good is an increasing function of the total number of labelled products the firm has in its product line.

From equation (10) we can derive a threshold value $\bar{n}_{1}$ for which it is true that $\rho(H, q, n, \bar{c})=1 \forall n \geq \bar{n}_{1}$. Consumers observing a number of labelled

\footnotetext{
${ }^{8}$ The event $c=0$ occurs with a probability of 0 , so removing this single point does not change the distribution. Therefore, we can concentrate our analysis on $\rho(H, q, n, \bar{c}) \neq 0$.

${ }^{9}$ From equation (9) we get
}

$$
\rho(H, q, n, \bar{c})=\left\{\begin{array}{cll}
0 & \text { if } & A=0 \\
\left.\frac{\mu^{\star} H}{\bar{c}}\left(1-(1-q)^{n}\right)\right) & \text { if } & 0<A<\bar{c} \\
1 & \text { if } & A \geq \bar{c}
\end{array}\right.
$$

${ }^{10}$ Since $\mu$ is an increasing function of $n$. 
products $n \geq \bar{n}_{1}$, and no detection of bad labels, know that they are being offered high quality products with a probability $\rho(H, q, n, \bar{c})=1$. We find

$$
\bar{n}_{1}=\frac{\ln (1-\bar{c} / H)}{\ln (1-q)}
$$

The consumers can be sure about getting high quality products, meaning that $\rho(H, q, n, \bar{c})=1$, only if even the least efficient firm type, i.e., the one with $c=\bar{c}$, satisfies condition (8). For any number of labelled products smaller than the threshold value, consumers have beliefs $\mu^{\star}<1$ that they get high quality and are willing to pay the corresponding price.

Proposition 2 summarizes the equilibrium if cost is not observable.

Proposition 2 Given the above defined consumer behavior and that $c$ is unknown to the consumers, we find the following Bayesian equilibrium:

- if consumers observe $n \geq \bar{n}_{1}$ as defined in equation (11) and no detection of a bad label, they expect only high quality products $\left(\mu^{\star}=1\right)$ and pay the full premium $H$ per unit of the good. A monopolist with $n \geq \bar{n}_{1}$ will decide to produce only high quality products and sets the price $P(H, q, c, n, \mu)=H$.

- if consumers observe that $n<\bar{n}_{1}$ and no detection of a bad label, they expect being offered high quality with a probability of $\mu^{\star}<1$. Thus, they are willing to pay a price of $\mu^{\star} H$ per unit of the good. A monopolist with $n<\bar{n}_{1}$ will decide to produce high or low quality products depending on its cost $c$ and sets the price $P(H, q, c, n, \mu)=\mu^{\star} H$.

- if consumers observe the detection of at least one bad label, they expect only low quality products and don't pay any premium. A monopolist who suffered the detection of a bad label will set the price $P(\cdot)=0$.

Comparing $\bar{n}_{1}$ with the threshold value found in equation (4) where consumers know the firm's cost, we see that $\bar{n}_{1} \geq \bar{n}$ since 


$$
\frac{\ln (1-\bar{c} / H)}{\ln (1-q)} \geq \frac{\ln (1-c / H)}{\ln (1-q)}
$$

For all but the least efficient firm type the threshold value is larger when consumers cannot observe $c$. Thus, the least efficient firm type, i.e., the type with the highest possible production $\operatorname{costs} c=\bar{c}$, sets the limit above which consumers completely trust in being offered high quality.

Comparing the situations where $c$ is/is not observable we can distinguish three cases:

- $n \geq \bar{n}_{1} \geq \bar{n}$ : If the firm's number of labelled products $n$ lies above the threshold value in both cases, it will produce high quality and set the price equal to $H$ which consumers are willing to pay if there is no detection. For those types of firms there is no difference regardless of $c$ being observable or not.

- $\bar{n}_{1}>n \geq \bar{n}$ : Such a firm will produce high quality and set price equal to $H$ if $c$ is observable. If $c$ is not observable it will either produce high or low quality, depending on its cost, ${ }^{11}$ and set price equal to $\mu^{\star} H$. Those types of firms that produce high quality in both situations will receive less if $c$ is not observable. For types of firms producing high quality if $c$ is observable, but low quality if $c$ is not observable it is ambiguous in which situation they do better. ${ }^{12}$

$\cdot \bar{n}_{1} \geq \bar{n}>n$ : In this case the firm will produce low quality regardless of $c$

\footnotetext{
${ }^{11}$ The condition to produce high quality if $c$ is observable is $\left(1-(1-q)^{n}\right) H \geq c$. This being satisfied does not imply that $\left(1-(1-q)^{n}\right) \mu^{\star} H \geq c$, the analogous condition if $c$ is not observable, is also satisfied.

${ }^{12}$ If cost is known to consumers the firm's profit is $n(H-c)$ whereas it is $n \mu^{\star} H$ if $c$ is unobservable.
} 
being observable or not. ${ }^{13}$ This type of firm will benefit from the information asymmetry with respect to cost, since it will receive 0 for its low quality products if $c$ is observable but $\mu^{\star} H$ if $c$ is not observable.

These findings are summarized in the following lemma.

Lemma 3 Assuming a uniform distribution for the cost $c$, we find that a firm with a large number of labelled products will make the same profit whether $c$ is observable for consumers or not. Efficient types of firms with a medium number of labelled products tend to do worse, while inefficient types of firms with a small number of labelled products benefit from the information asymmetry with respect to $c$.

\section{Conclusion}

In this paper we have considered a market for labelled credence goods in which a monopolist faces a number of homogeneous consumers. The monopolist decides whether to produce high or low quality goods. This quality is not directly observable by the consumers but may be found out by means of watchdog agencies, information leaks etc. Assuming that consumers trust a firm as a whole, resp. its labels, rather than single products, we designed a model in which consumers reduce the price they are willing to pay for a labelled good if they found out that the firm tried to cheat them by offering low quality products under a label indicating high quality. Given this consumer behavior we find that, regardless of $c$ being observable or not, we can derive a threshold value for the number of labelled products, above which consumers can be sure of getting high quality. If the number of labelled products is

\footnotetext{
${ }^{13}$ Notice that $\left(1-(1-q)^{n}\right) H<c$ implies that $\left(1-(1-q)^{n}\right) \mu^{\star} H<c$.
} 
below the threshold value and $c$ is not observable, then the consumers derive the probability of being offered high quality products. This probability, and therefore the price consumers are willing to pay, is increasing with the number of labelled products, assuming a uniform distribution of $c$. The number of labelled products thus serves as a signal for the quality of the labelled credence goods. We also saw that firms with a small number of labelled goods and high costs tend to benefit from the information asymmetry with respect to cost whereas medium sized firms, measured by the number of goods, with low costs tend to do worse if $c$ is not observable. 


\section{References}

CABRAL, L.M.B. "Stretching Firm and Brand Reputation." RAND Journal of Economics, Vol. 31, No. 4 (2000), pp. 658-673.

CHOI, J.P. "Brand Extension as Informational Leverage." Review of Economic Studies, Vol. 65 (1998), pp. 655-669.

DULLECK, U. AND KERSCHBAMER, R. "On Doctors, Mechanics and Computer Specialists Or Where are the Problems with Credence Goods?" Working Paper University of Vienna, No. 0101 (2001).

EMONS, W. "Credence Goods and Fraudulent Experts." RAND Journal of Economics, Vol. 28 (1997), pp. 107-119.

EMONS, W. "Credence Goods Monopolists." International Journal of Industrial Organization, Vol. 19 (2001), pp. 375-389.

GOLAN, E., KUCHLER, F., AND MITCHELL L. "Economics of Food Labeling." Journal of Consumer Policy, Vol. 24, No. 4 (2001), pp. 117-184.

SHAPIRO, C. "Consumer Information, Product Quality, and Seller Reputation." RAND Journal of Economics, Vol. 13, No. 1 (1982), pp. 20-35.

SHAPIRO, C. "Premiums for High Quality Products as Returns to Reputation." Quarterly Journal of Economics, Vol. 98, No. 4 (1983), pp. 659-680. SULLIVAN, M. "Measuring Image Spillovers in Umbrella-branded Products." Journal of Business, Vol. 63, No. 4 (1990), pp. 309-329.

THØGERSEN, C. "Psychological Determinants of Paying Attention to EcoLabels in Purchase Decisions: Model Development and Multinational Validation." Journal of Consumer Policy, Vol. 23, No. 4 (2000), pp. 285-313.

WERNERFELT, B. "Umbrella Branding as a Signal of New Product Quality: An Example of Signalling by Posting a Bond." RAND Journal of Economics, Vol. 19, No. 3 (1988), pp. 458-466. 


\section{Consumer}

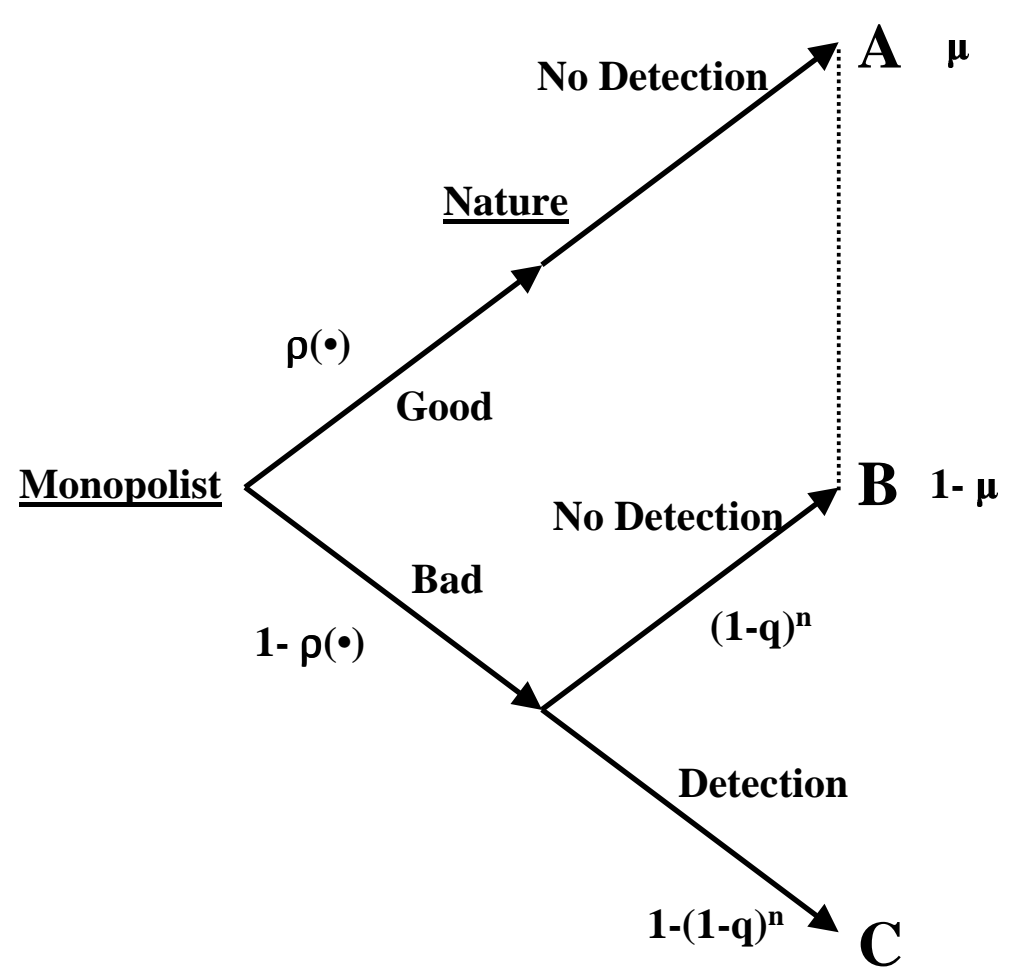

Figure 1: $\quad$ Game tree if $c$ is unknown to consumers. 